adult service, 1 patient had been discharged and 2 patients did not attend. Adherence was optimal in 10 (44.5\%), suboptimal in $9(40.9 \%)$ and poor in $3(13.64 \%)$ patients (see figure 1). Their mean BEC was $0.35 \times 10^{9} / 1( \pm 0.33)$, FeNO 48.7 ppb $( \pm 38.1)$ and FEV1\% was $86.8 \%( \pm 12.2)$.

Conclusion While there was an incremental improvement in adherence during the first year of adult care with resultant improvements in asthma biomarkers, on-going support is needed to further increase medicines use and to ensure this behaviour persists.

\section{S35 CREATING BEHAVIOURAL PERSONAS TO DRIVE BETTER DESIGN IN HEALTH TECHNOLOGY FOR ASTHMA SELF- MANAGEMENT}

${ }^{1} \mathrm{~A}$ Fallas, ${ }^{1} \mathrm{~A}$ Cumella, ${ }^{1} \mathrm{~A}$ Whittamore, ${ }^{1} \mathrm{~B}$ West, ${ }^{1} \mathrm{C}$ Cheung, ${ }^{1} \mathrm{~J}$ Shopland, ${ }^{2} \mathrm{~A}$ Herbec, ${ }^{2} \mathrm{C}$ Stefanidou, ${ }^{2} \mathrm{~L}$ Porter, ${ }^{2} \mathrm{~N}$ Gold, ${ }^{2} \mathrm{P}$ Lacey, ${ }^{2} \mathrm{P}$ Bondaronek, ${ }^{2} \mathrm{~T}$ Papakonstantinou, ${ }^{2} \mathrm{~V}$ Mallion, 'S Walker. ${ }^{1}$ Asthma UK and British Lung Foundation, London, UK; ${ }^{2}$ PHE Behavioural Insights Team, Public Health England, London, UK

\subsection{6/thorax-2021-BTSabstracts.41}

Introduction and Objectives The health burden from asthma can be reduced through better provision of basic care and better self-management. Most health technology products tend to target a narrow range of behaviours with limited behaviour change techniques (BCTs), take a homogeneous approach towards the diverse population of people with asthma and have poor uptake.

Objective to identify and characterise distinct behavioural self-management archetypes among UK adults with asthma with the aim of creating behavioural personas that can be used by product developers to better address the needs of people with asthma.

Methods We conducted a scoping review of grey and academic literature, followed by workshops with subject matter experts to identify key behaviours and influences relevant to asthma self-management. We then conducted a rapid review and behavioural analysis on these key behaviours which were then synthesised into a behavioural systems map. A survey was constructed to explore a subset of key behaviours and influences in more detail including asthma management, asthma control, inhaler use, support seeking, monitoring, and technology use. The survey was administered to 2,324 people reflective of the UK adult asthma population. The results were analysed and synthesised using mixed methods. Data were segmented using Multiple Correspondence Analysis and k-means cluster analysis, and further statistical analysis was performed to identify factors independently associated with adherence behaviour. The results were synthesised into behavioural personas that characterise people with optimal vs. suboptimal preventer inhaler adherence in behavioural terms, alongside relevant design prompts and suggested BCTs.

Results Segmenting by inhaler use revealed behaviours distributed as shown in figure 1 . Segmenting by adherence to preventer-type inhalers alone revealed pronounced differences between optimal and sub-optimal behaviour clusters in terms of age and behavioural factors (including: skills, decision making, behavioural regulation, environmental opportunities, attitudes, motives, intentions, beliefs, identity, and emotions).

Conclusions We have developed unique insight into behaviours of people with asthma and the influences on these behaviours.

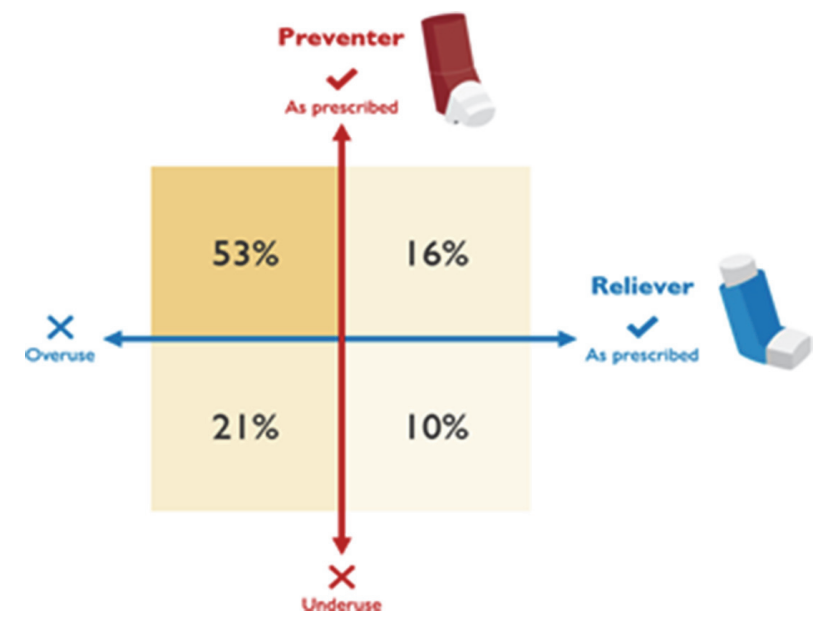

\section{Abstract S35 Figure 1}

We believe this work can contribute to a paradigm shift in the design of asthma health technology products, towards targeting new behaviours and their influences for change and ultimately driving better self-management and fewer asthma deaths.

\section{S36 PRESCRIBING PATTERNS AND TREATMENT ADHERENCE IN PATIENTS WITH ASTHMA DURING THE COVID-19 PANDEMIC}

${ }^{1} \mathrm{H}$ Dhruve, ${ }^{1} \mathrm{~J}$ Dhariwal, ${ }^{1} \mathrm{G}$ d'Ancona, ${ }^{2} \mathrm{~S}$ Holmes, ${ }^{1} \mathrm{~A}$ NanzerKelly, ${ }^{1} \mathrm{DJ}$ Jackson. ${ }^{1}$ Guys and St Thomas, London, UK; ${ }^{2}$ Park Medical Practice, Somerset, UK

\subsection{6/thorax-2021-BTSabstracts.42}

Introduction and Objective The COVID-19 pandemic has witnessed a reduction in asthma exacerbations in the UK. Several factors may underpin this, including reduced transmission of seasonal viruses and improved use of or adherence to inhaled corticosteroids (ICS). This study aims to investigate whether ICS use has changed during the pandemic for patients with asthma.

Methods Using the OpenPrescribing database, we analysed prescribing patterns of ICS, salbutamol and peak flow meters from January 2019 to January 2021 across England. Additionally, using a sample asthma cohort from 3 primary care practices, we assessed individual prescription patterns and ICS adherence across the two-year period. ICS adherence has been defined according to the medication possession (MPR) ratio: good $(\geq 75 \%)$, sub-optimal $(50-74 \%)$, poor $(25-49 \%)$ and non-adherence $(<25 \%)$.

Results A sharp increase in national ICS prescriptions was observed at the start of the pandemic in March 2020 representing a 50\% increase compared to February 2020. Thereafter national ICS prescription rates appear to have returned to normal levels. The sample asthma cohort included 1132 patients (762 patients treated with ICS across 2019 and 2020). Overall, adherence to ICS improved in 2020 $(P<0.001)$, with the proportion of patients meeting 'good adherence' $(\geq 75 \%)$ increasing from $34 \%$ to $42 \%(P<0.001)$. Analysis of this cohort suggested the March 2020 spike predominantly reflected improved adherence rather than a hoarding effect of multiple inhalers or new prescriptions for ICSnaïve individuals. Increasing age was associated with higher 
levels of ICS adherence. A similar spike in salbutamol occurred in March 2020, however, an overall reduction in salbutamol prescriptions was seen in $2020(P=0.039)$. National figures highlighted a progressive increase in prescription of peak flow meters over 2020.

Conclusion A marked spike in national ICS prescriptions occurred in March 2020. This increase appears to reflect improved adherence in patients with low levels of adherence rather than a hoarding effect or large-scale initiation in ICSnaïve patients. Despite a comparable spike in salbutamol prescriptions, 2020 saw an overall reduction in salbutamol prescriptions. Prescription of peak flow meters steadily increased over 2020 in keeping with the need for more remote monitoring.

\section{Beyond acid-fast: diagnosis and treatment of TB in the 21st Century}

\section{S37 DUAL STEP INTERFERON-GAMMA RELEASE ASSAY TESTING CAN IMPROVE TUBERCULOSIS (TB) RISK STRATIFICATION IN CONTACTS OF PULMONARY TB: A PROSPECTIVE ADULT HOUSEHOLD CONTACT COHORT STUDY}

${ }^{1} \mathrm{JW}$ Kim, ${ }^{1 \mathrm{~J}}$ Nazareth, ${ }^{2} \mathrm{~J}$ Lee, ${ }^{2} \mathrm{G}$ Woltmann, ${ }^{3} \mathrm{~A}$ O'Garra, ${ }^{2} \mathrm{R}$ Verma, ${ }^{1} \mathrm{P}$ Haldar. ${ }^{1}$ University of Leicester, Leicester, UK; ${ }^{2}$ University Hospitals of Leicester NHS Trust, Leicester, UK; ${ }^{3}$ Francis Crick Institute, London, UK

10.1136/thorax-2021-BTSabstracts.43

Introduction Studies report modest and variable predictive value of an interferon-gamma release assay (IGRA) test performed 8-12 weeks after index notification, to identify latent tuberculosis (TB) infection at risk of progressing to active TB. There is limited data evaluating predictive value of changes in the IGRA response with serial testing following recent exposure in a low-TB burden setting.

Objectives To quantify the risk of progression to active TB using the serial IGRA response between baseline and 3 months in pulmonary $\mathrm{TB}$ contacts.

Methods We performed an ethically approved prospective cohort study of pulmonary TB contacts between September 2015 and May 2018. Participants were recruited immediately after index case notification and had IGRA (QuantiFERON-TB Gold, QFT) test at baseline and 3 months. QFT+ve contacts did not receive chemoprophylaxis, but were followed prospectively up to 4 years with three monthly review during the first 2 years. In contacts developing TB (progressors), whole genome sequencing (WGS) was performed to inform case linkage. We defined changes in serial IGRA response as conversion (QFT negative to positive); or in contacts QFT+ve at baseline, as $>0.27 \mathrm{IU} / \mathrm{L}$ change in the QFT response, representing $>2$ standard deviations of the mean serial QFT variability observed in a control group without recent TB exposure.

Results 297 contacts were followed for a median of 1437 days (IQR 1159-1460). 124 contacts (41.8\%) were QFT+ve at 3 months, of which 19 seroconverted from baseline. 20 progression events occurred and 6 diagnoses (30\%) were made within 3 months of index notification including two QFT-ve cases. The remaining 14 cases were QFT+ve at baseline, and diagnosed after a median of 285 days. All 9 culture confirmed progressors were WGS matched to their index. For
Abstract S27 Table 1 Two -year incident tuberculosis risks in untreated adults pulmonary TB contacts

\begin{tabular}{ll}
\hline & 2- year risk $(95 \% \mathrm{Cl})$ \\
\hline QFT positive (at 3 month) & $10.8(4.8-16.5)$ \\
Quantitative QFT (at 3 month) & $12.3(0-24.5)$ \\
$<1$ & $7.5(0-17.0)$ \\
$1-4$ & $11.7(0.0-19.5)$ \\
$>4$ & \\
Serial QFT & $33.6(7.5-52.3)$ \\
Seroconversion & \\
If QFT positive at baseline & $6.3(0-14.5)$ \\
Significant increase & $2.9(0-8.5)$ \\
Significant decrease & $8.8(0-35.8)$ \\
No significant change & \\
\hline
\end{tabular}

QFT+ve contacts, 2-year risk of incident TB was 10.8\%, with no significant difference according to index smear status or quantitative QFT value (Table). Serial QFT identified greatest risk in seroconverters (2-year risk $=33.6 \%$ ) but no increased risk in QFT+ve contacts with a significant change after 3 months (table 1).

Conclusions Our data suggests serial QFT testing at baseline and 3 months after index notification improves risk stratification in pulmonary $\mathrm{TB}$ contacts.

Please refer to page A188 for declarations of interest related to this abstract.

\section{S38 EVALUATION OF MYCOBACTERIUM TUBERCULOSIS- SPECIFIC IFN-G, TNF-A, CXCL10, IL2, CCL2, CCL7 AND CCL4 LEVELS FOR ACTIVE TUBERCULOSIS DIAGNOSIS}

${ }^{1} \mathrm{~A}$ Fries, ${ }^{2} \mathrm{~V}$ Mandagere, ${ }^{3} \mathrm{R}$ Parker, ${ }^{3} \mathrm{M}$ Tolosa-Wright, ${ }^{3} \mathrm{~L}$ Berrocal-Almanza, ${ }^{3} \mathrm{~L}$ Hoang ${ }^{3}$ A Boakye, ${ }^{4} \mathrm{~A}$ Halliday, ${ }^{3} \mathrm{~A}$ Lalvani. ' Nuffield Department of Medicine, University of Oxford, Oxford, UK; ${ }^{2}$ Bristol Medical School, University of Bristol, Bristol, UK; ${ }^{3}$ Tuberculosis Research Centre, National Heart and Lung Institute, Imperial College London, London, UK; ${ }^{4}$ Cellular and Molecular Medicine, University of Bristol, Bristol, UK

\subsection{6/thorax-2021-BTSabstracts.44}

Background Novel diagnostic tests for active tuberculosis (ATB) are urgently needed. We aimed to efficiently and robustly assess whether seven previously identified, promising biomarkers (IFN-g, TNF-a, CXCL10, IL2, CCL2, CCL7 and CCL4) could distinguish patients with ATB within a cohort of patients presenting with the full clinical spectrum of suspected $\mathrm{TB}$ in routine practice.

Methods We designed a nested case-control study $(n=92)$ within the IDEA study. ${ }^{1}$ Uniquely, we enriched our ATB population to include $\sim 50 \%$ patients in whom current IGRAs fail (and unmet clinical need is greatest), to assess whether any biomarker offered superior diagnostic accuracy to IFN-g. We utilised stored supernatants from QFT-GIT tests performed in the IDEA study and compared Mycobacterium tuberculosis-specific biomarker levels in patients with ATB and non-tuberculosis respiratory diseases using Meso Scale Discovery U-PLEX assays. We analysed group differences using Kruskal-Wallis tests.

Results In phase I, we analysed IFN-g, TNF-a, CXCL10, IL2, CCL2, CCL7 and CCL4 levels in 32 patients. MSD-measured biomarkers (except CCL4) detected higher numbers of true positives (TP) compared to QFT-GIT, however, all biomarkers 